\title{
Disrupted balance of long and short-range functional connectivity density in Alzheimer's disease (AD) and mild cognitive impairment (MCl) patients: a resting-state fMRI study
}

\author{
Yanping Mao ${ }^{1 \#}$, Zhengluan Liao ${ }^{2 \#}$, Xiaozheng $\mathrm{Liu}^{3}$, Ting $\mathrm{Li}^{4}$, Jiaojiao $\mathrm{Hu}^{1}$, Dansheng Le ${ }^{5}$, Yangliu Pei ${ }^{6}$, \\ Wangdi Sun ${ }^{5}$ Jixin Lin ${ }^{7}$, Yaju Qiu ${ }^{2}$, Junpeng Zhu' ${ }^{2}$, Yan Chen ${ }^{2}$, Chang Qi ${ }^{2}$, Heng Su ${ }^{2}$, Enyan Yu ${ }^{1}$ \\ ${ }^{1}$ Department of Clinical Psychology, Cancer Hospital of the University of Chinese Academy of Sciences, Zhejiang Cancer Hospital, Hangzhou, \\ China; ${ }^{2}$ Department of Psychiatry, Zhejiang Provincial People's Hospital, People's Hospital of Hangzhou Medical College, Hangzhou, China; \\ ${ }^{3}$ Department of Radiology of the Second Affiliated Hospital and Yuying Children's Hospital, Wenzhou Medical University, Wenzhou, China; \\ ${ }^{4}$ Medical Department, Qingdao University, Qingdao, China; ${ }^{5}$ The Second school of Medical, Zhejiang Chinese Medical University, Hangzhou, \\ China; ${ }^{6}$ Graduate Department, Bengbu Medical College, Bengbu, China; ${ }^{7}$ Department of Internal Medicine, Shengsi County People's Hospital, \\ Zhoushan, China \\ Contributions: (I) Conception and design: Y Mao, Z Liao, E Yu; (II) Administrative support: Z Liao, E Yu; (III) Provision of study materials or \\ patients: Y Mao, Z Liao, Y Qiu, J, Zhu, Y Chen, C Qi, Y Tan, H Su; (IV) Collection and assembly of data: Y Mao, Z Liao; (V) Data analysis and \\ interpretation: Y Mao, X Liu; (VI) Manuscript writing: All authors; (VII) Final approval of manuscript: All authors. \\ \#These authors contributed equally to this work. \\ Correspondence to: Enyan Yu. Department of Clinical Psychology, Cancer Hospital of the University of Chinese Academy of Sciences, Zhejiang \\ Cancer Hospital, Hangzhou, China. Email: yuenyan@aliyun.com.
}

Background: Alzheimer's disease (AD) is an age-progressive neurodegenerative disorder that affects cognitive function. There have been several functional connectivity (FC) strengths; however, FC density needs more development in $\mathrm{AD}$. Therefore, this study wanted to determine the alternations in resting-state functional connectivity density (FCD) induced by Alzheimer's and mild cognitive impairment (MCI).

Methods: One hundred and eleven AD patients, 29 MCI patients, and 73 healthy controls (age- and sexmatched) were recruited and assessed using resting-state functional magnetic resonance imaging (MRI) scanning. The ultra-fast graph theory called FCD mapping was used to calculate the voxel-wise short- and long-range FCD values of the brain. We performed voxel-based between-group comparisons of FCD values to show the cerebral regions with significant FCD alterations. We performed Pearson's correlation analyses between aberrant functional connectivity densities and several clinical variables with adjustment for age and sex.

Results: Patients with cognition decline showed significantly abnormal long-range FCD in the cerebellum crus I, right insula, left inferior frontal gyrus, left superior frontal gyrus, left inferior frontal gyrus, and right middle frontal gyrus. The short-range FCD changed in the cerebellum crus I, left inferior frontal gyrus, left superior occipital gyrus, and right middle frontal gyrus. The long- and short-range functional connectivity in the left inferior frontal gyrus was positively correlated with Mini-mental State Examination (MMSE) scores. Conclusions: FCD in the identified regions reflects mechanism and compensation for loss of cognitive function. These findings could improve the pathology of AD and MCI and supply a neuroimaging marker for $\mathrm{AD}$ and MCI.

Keywords: Functional connectivity density (FCD); Alzheimer's disease (AD); mild cognitive impairment (MCI)

Submitted Jul 31, 2020. Accepted for publication Dec 02, 2020.

doi: $10.21037 /$ atm-20-7019

View this article at: http://dx.doi.org/10.21037/atm-20-7019

\footnotetext{
^ ORCID: 0000-0002-4087-4568.
} 


\section{Introduction}

Alzheimer's disease (AD) is an age-progressive neurodegenerative disorder that may cause cognitive function impairment even in its prodromal stage termed as mild cognitive impairment (MCI) (1). Cognitive dysfunction, which is the most common $\mathrm{AD}$ symptom, is characterized by dramatic and dangerous behaviors that mainly contributed to $\mathrm{AD}$-associated mortality and burden (2). Although there has been significant progress in elucidating the mechanisms underlying $\mathrm{AD}$, they remain unclear (3).

Advances in neuroimaging techniques have allowed more efficient and noninvasive AD assessment (4-6). Besides, cognitive function scale can be used to early diagnosis in clinical and some blood markers and metabolites predict the development of $\mathrm{AD}$ in some research.

Morphometric studies using magnetic resonance imaging (MRI) have reported a significant reduction in the gray matter density of the hippocampus in patients with $\mathrm{AD}$ (4). Further, diffusion tensor imaging has shown that anisotropy in the cingulate and mean diffusivity in the hippocampus are $\mathrm{AD}$ progression predictors (7). A method called functional connectivity (FC) can measure spontaneous brain function (8), which indicates the temporal correlation of spontaneous brain activity fluctuations between spatially distinct regions. Several studies have reported abnormal FC in the bilateral hippocampal functional network (9).

A functional connectivity density (FCD) can measure the number of functional connections of the target voxel with the other voxel (10). A higher FCD value shows that the voxel is functionally connected to a large voxel number. It suggests that the voxel may be more crucially involved in information processing than voxels with lower FCD values.

Studies have used long- and short-range FCD to investigate the abnormal brain FC in borderline personality disorder (11), sleep deprivations (12), alcohol abuse (10), and autism spectrum disorder (13). Calculating the shortrange FCD can determine the function of the special voxels in functionally specialized systems, while long-range FCD is more exact for showing the functional integration of wholebrain networks.

This study aimed to identify differences in the spontaneous functional organization by using long- and short-range $\mathrm{FCD}$ values in many patients with $\mathrm{AD}$ and MCI and normal controls (NC). The within-group correlation analysis revealed the relationship between cognitive function and long- and short-range FCD in the identified abnormal regions. We present the following article in accordance with the MDAR reporting checklist (available at http://dx.doi.org/10.21037/atm-20-7019).

\section{Methods}

\section{Participants}

This study recruited 140 cognitive impairment patients, including $111 \mathrm{AD}$ patients and $29 \mathrm{MCI}$ patients from September 2016 to December 2018 at Zhejiang Provincial People's Hospital. The control group was healthy, ageand sex-matched volunteers from the hospital. The Ethics Committee of Zhejiang Provincial People's Hospital authorized the study protocol (No. 2012KY002) and was performed following the Declaration of Helsinki (as revised in 2013). Informed consent was taken from all patients.

We collected social characteristics, including sex, age, educational status, and disease conditions. The hematological examination, neuropsychological assessment, and other medical information were collected. The Minimental State Examination (MMSE) and Montreal Cognitive Assessment scale (MoCA) were the most common scales for cognitive function assessment. All the patients were aged between 55 and 85. The diagnostic standard of AD met the National Institute of Neurologic and Communicative Disorders and Stroke and the AD and Related Disorders Association criteria, MMSE score $\leq 24$ and MoCA score $\leq 26$. Patients with MCI were determined using the following criteria: impaired memory complaints, maintaining normal performance, and MMSE scores of $>24$ and $\geq 27$. The inclusion criteria for NC were: age- and sex-matched volunteers without cognition impairment, the MMSE score $\geq 28$. The exclusion criteria: other dementia and involving memory disorder, the psychiatric illness which may cause the dementia symptom, a history of alcoholism or drug abuse, and any organic brain disease. Finally, we enrolled 111 patients with $\mathrm{AD}, 29$ patients with MCI, and $73 \mathrm{NC}$.

\section{MRI acquisition}

We acquired all MRI data using a 3.0-T MR scanner (GE Healthcare, Discovery MR750, United States). The head is fixed with sponge pads to avoid head motion artifacts and scanner noise. All participants were ordered not to fall asleep while keeping their eyes closed during the scan to keep brain oxygen level at stable value. The structural MRIs were acquired using a high-resolution three- 
dimensional T1-weighted magnetization-prepared rapid gradient echo (MPRAGE) sagittal sequence with scanning parameters of repetition time $(\mathrm{TR})=6.7 \mathrm{~ms}$, echo time (TE) $=2.9 \mathrm{~ms}$, inversion time $(\mathrm{TI})=450 \mathrm{~ms}$, slice thickness/ gap $=1 / 0 \mathrm{~mm}, \mathrm{FOV}=256 \times 256 \mathrm{~mm}^{2}$, flip angle $=12^{\circ}$, matrix $=256 \times 256$; there were 192 sagittal slices collected from each subject. A resting-state fMRI (rs-fMRI) scan was performed an echo planar imaging (EPI) sequence to collect the images with the following parameters: repetition time/echo time $(\mathrm{TR} / \mathrm{TE})=2,000 / 30 \mathrm{~ms}$, slice thickness $=3.2 \mathrm{~mm}$ and the gap $=0 \mathrm{~mm}$, the field of view $(\mathrm{FOV})=220 \times 220 \mathrm{~mm}^{2}$, and the flip angle $=90^{\circ}$. Each acquisition had 210 timepoints that had 44 slices.

\section{Functional image preprocessing}

Data Processing \& Analysis for Brain Imaging (DPABI) toolbox was used to preprocess the re-fMRI data (14). We discarded the first ten points of each participant to keep the signal to reach equilibrium and saturation effects. The remaining 200 consecutive timepoints were corrected for slice timing to the first timepoint for head motion correction. The head motion correction and spatial normalization were set to the standard EPI temple with a resampled voxel size of $3 \times 3 \times 3 \mathrm{~mm}^{3}$. Participants were included with a head motion of less than $3 \mathrm{~mm}$ and $3^{\circ}$. Then, the fMRI data were detrended to remove the linear detrending and band-pass filtering $(0.01-0.08 \mathrm{~Hz})$. Finally, using the nuisance covariates reduced head motion, global signal, cerebrospinal fluid, and white matter signal.

\section{Long- and short-FCD calculation}

After preprocessing the fMRI data, the long- and shortrange FCD of the special voxels were calculated using a graph theoretical network analysis toolbox v1.2.1 (GRATNA v1.2.1) (15). A voxel was used to reveal the association of the time course of the other voxels by Pearson's correlation. The strength of the functional connections of a given voxel described the node degree in the binary graph. The detailed calculation process of the local and global FCD has been described (16). The connectional FCD threshold was set as 0.6 (17). The actual physical distance of betweenregion connections has been reported to be approximately $75 \mathrm{~mm}$ (18). FC between a given voxel and other voxels exceeding and within the actual physical distance was defined as long- and short-range FCD, respectively. For further data analysis, the long- and short-range FCD maps were converted to $Z$ scores (19). Finally, these maps were smoothed with a $6-\mathrm{mm}$ full-width at half-maximum Gaussian kernel using SPM8 (https://www.fil.ion.ucl.ac.uk/ spm/software/spm8/).

\section{Statistical analysis}

SPSS 26.0 (SPSS Inc., Chicago, IL, USA) was used to analyze demographic and clinical variables. We analyzed variance to determine differences in age, education level, MMSE, and MoCA. The sex ratio was compared with the $\chi^{2}$ test. Statistical significance was set as $\mathrm{P}<0.05$. Analysis of covariance (ANOVA) was performed using DPABI toolbox v4.0 to examine among-group differences (20). Then, based on the brain mask from the abnormal brain regions of the ANOVA, post hoc analysis based on a two-sample $t$-test is used to perform two groups by comparison. AlphaSim corrections were used for multiple comparisons after removing the confounding effects of age, sex, and relative root mean square of head motion by regression. The significance level was set at an uncorrected $\mathrm{P}<0.005$, with a cluster size $>25$, which corresponded to a corrected $\mathrm{P}<0.05$ for each voxel. The mean $\mathrm{z}$ values of long- and short-range FCD were extracted from the cluster with abnormal FCD to explore the correlation with the cognition function assessment. Pearson correlation coefficients were calculated between these variables after assessing the normality of this data to reveal the strength of the relationship.

\section{Results}

\section{Demographic and clinical features}

Table 1 shows the demographic and clinical characteristics of the AD, MCI, and NC groups. There were no significant group differences in sex, age, and education. However, there was a significant difference in MMSE and MoCA scores.

\section{Abnormal short-range FCD}

The analysis of variance was used to reveal the difference in short-range FCD in different cognition decline groups at select brain regions like the cerebellum crus I, left inferior frontal gyrus, left superior occipital gyrus, and right middle frontal gyrus. Short-range FCD was found to increase left cerebellum crus 1 and right middle frontal gyrus in $\mathrm{AD}$ patients. Short-range FCD increased in left superior occipital gyrus in MCI patients. Compared with MCI patients, $\mathrm{AD}$ 
Table 1 Demographic and clinical characteristics of the participants

\begin{tabular}{lcccc}
\hline Characteristics & $\mathrm{AD}(\mathrm{n}=111)$ & $\mathrm{MCl}(\mathrm{n}=29)$ & $\mathrm{NC}(\mathrm{n}=73)$ & $32: 41$ \\
\hline Sex & $37: 74$ & $13: 16$ & $66.30 \pm 9.52$ & 0.273 \\
Age & $68.28 \pm 9.64$ & $65.90 \pm 10.05$ & $8.26 \pm 3.38$ & 0.284 \\
Education & $7.87 \pm 4.41$ & $8.45 \pm 4.60$ & $28.77 \pm 0.83$ & 0.723 \\
MMSE & $17.24 \pm 5.57$ & $25.97 \pm 0.94$ & $27.19 \pm 1.66$ & $<0.001$ \\
MOCA & $13.39 \pm 6.30$ & $21.21 \pm 3.83$ & $<0.001$ \\
\hline
\end{tabular}

$\chi^{2}$-test was used in sex, analysis of variance in age, education level, MMSE, and MoCA. AD, Alzheimer's disease; MCl, mild cognitive impairment; NC, the normal control; MMSE, Mini-mental State Examination; MoCA, Montreal Cognitive Assessment.

Table 2 Regions showing abnormal short-range FCD in the AD group compared with the MCI group and NC group

\begin{tabular}{|c|c|c|c|c|c|c|}
\hline Brain regions & Voxels & BA & \multicolumn{3}{|c|}{ MNI coordinates } & $\mathrm{F} / \mathrm{T}$ value \\
\hline \multicolumn{7}{|l|}{ ANOVA } \\
\hline Cerebelum-Crus I-L & 48 & - & -36 & -48 & -36 & 7.7076 \\
\hline Frontal-Inf-Tri-L & 73 & 47 & -36 & 36 & 6 & 10.8696 \\
\hline Occipital-Sup-L & 25 & 19 & -12 & -78 & 42 & 10.1479 \\
\hline Frontal-Mid-R & 66 & 6 & 39 & -3 & 57 & 8.5307 \\
\hline \multicolumn{7}{|l|}{$\mathrm{AD}$ vs. $\mathrm{MCl}$} \\
\hline Frontal-Inf-Tri-L & 73 & 47 & -36 & 36 & 6 & -4.3604 \\
\hline Cerebelum-Crus I-L & 46 & - & -33 & -48 & -39 & 3.4949 \\
\hline Frontal-Mid-R & 56 & 8 & 30 & 6 & 57 & 3.7141 \\
\hline \multicolumn{7}{|l|}{$\mathrm{MCl}$ vs. $\mathrm{NC}$} \\
\hline Occipital-Sup-L & 25 & 19 & -12 & -78 & 42 & 4.0437 \\
\hline
\end{tabular}

FCD, functional connectivity density; AD, Alzheimer's disease; MCl, mild cognitive impairment; NC, normal control group; MNI, Montreal Neurological Institute; BA, Brodmann area; ANOVA, analysis of variance; L, left; R, right; Inf, inferior; Sup, superior; Mid, middle; Tri, triangular.

patients showed the significant attenuation of short-range FCD in the left inferior frontal gyrus (Table 2, Figure 1).

\section{Abnormal long-range FCD}

Long-range FCD had been found significant differences in the left cerebellum crus I, right insula, left inferior frontal gyrus, left superior frontal gyrus, and right middle frontal gyrus in distinct groups. Compared to the MCI group, there was a decreased long-range FCD found in the left inferior frontal gyrus and left superior frontal gyrus in $\mathrm{AD}$ patients. Compared with $\mathrm{NC}, \mathrm{AD}$ patients showed lower long-range FCD in the right insula, left inferior frontal gyrus, and right superior frontal gyrus. However, $\mathrm{AD}$ patients showed stronger long-range FCD in the left cerebellum crus I. Compared with the NC group, the MCI group showed lower long-range FCD in the right insula (Table 3, Figure 2). 


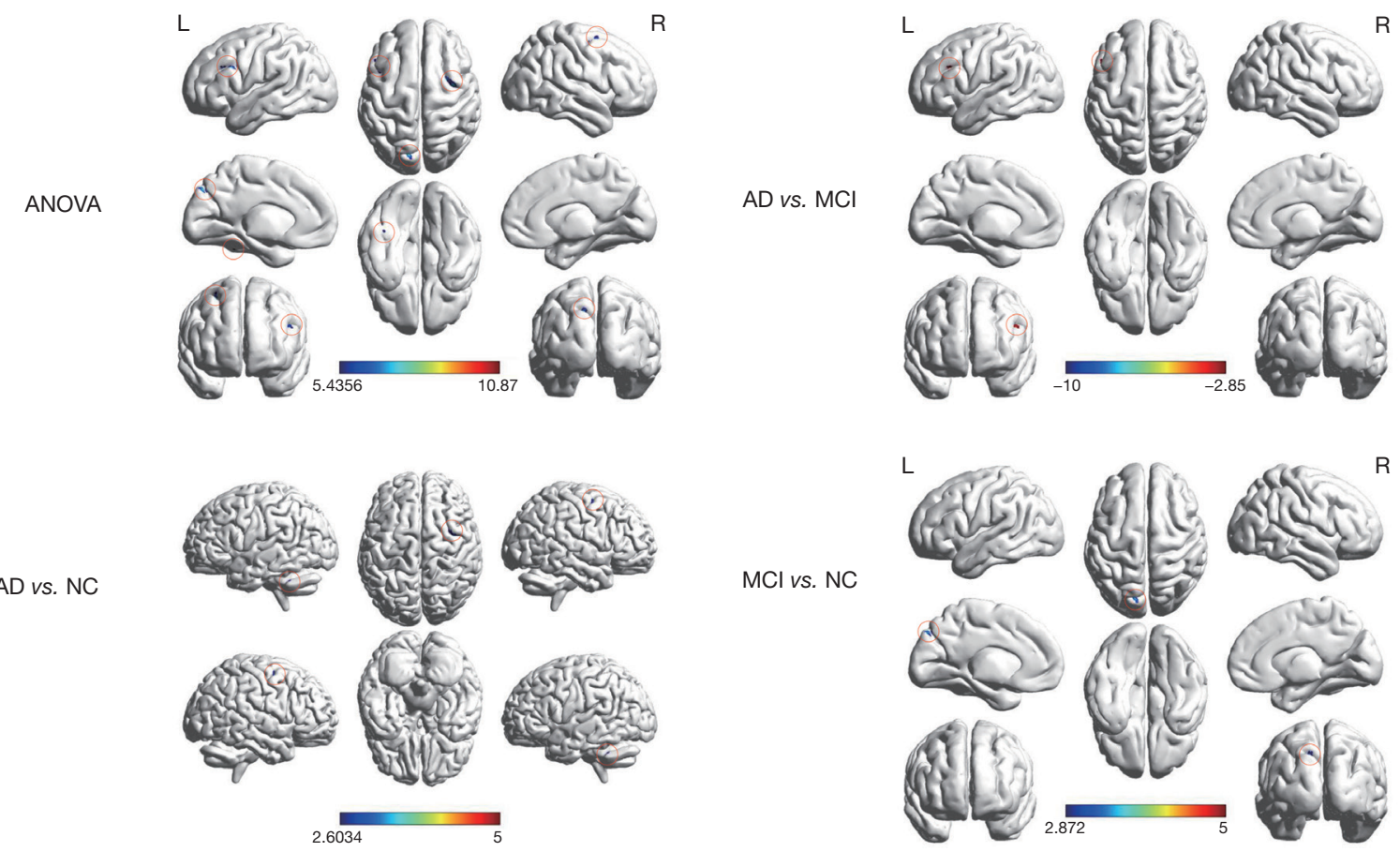

Figure 1 Spatial distribution of short-range FCD in the AD, MCI, and NC groups, as well as the statistical among-group differences. The among-group differences were corrected using AlphaSim $(\mathrm{P}<0.005$, a combined threshold of $\mathrm{P}<0.05$ with a minimum cluster size of 25 voxels). ANOVA, Analysis of variance; AD, Alzheimer's Disease; MCI, mild cognitive impairment; NC, normal control group; L, left; R, right; FCD, functional connectivity density.

\section{Correlation between abnormal FCD and cognitive function}

Positive correlations were confirmed between MMSE scores and the increased long-range FCD in the left inferior frontal gyrus. Further, the increased short-range FCD in the left inferior frontal gyrus was positively associated with MMSE scores (Figure 3). Other brain region FCD values showed no correlation to cognition function assessment.

\section{Discussion}

Our study is one of the first to find aberrant FCD may provide help in early diagnosis of cognitive decline patients. $\mathrm{AD}$ and MCI patients showed a abnormal long-range FCD in the cerebellum crus I, right insula, left inferior frontal gyrus, left superior frontal gyrus, left inferior frontal gyrus, and right middle frontal gyrus. There was a significant increased in the short-range FCD in the cerebellum crus I, left inferior frontal gyrus, left superior occipital gyrus, and right middle frontal gyrus. Further, there was a positive correlation of altered long-range FCD in the left inferior frontal gyrus with the MMSE scores, which showed impaired cognitive function. These findings suggest an association of altered brain function with specific brain areas showing disrupted intraregional and interregional interactions in individuals with cognition decline.

\section{Abnormal FCD in AD and MCI}

Our findings showed that the MCI and AD group had a abnormal short-range FCD and changed long-range FCD in the left inferior frontal gyrus, which is crucially involved in component processes of episodic memory and cognition (21). Given the findings above, the long-range FCD in the left inferior frontal gyrus in cognitive decline patients could imply the impaired memory, and the changed short-range FCD in the left inferior frontal gyrus in patients with $\mathrm{MCI}$ and $\mathrm{AD}$ could temporarily compensate for impaired memory (22). These findings could be applied for distinguishing individuals with abnormal cognition decline (23). Short-range FCD in MCI patients was higher than those in $\mathrm{AD}$ patients in the left inferior frontal gyrus. MCI is the early-stage of $\mathrm{AD}$ has 
Table 3 Regions showing abnormal long-range FCD in the AD group compared with the MCI group and NC group

\begin{tabular}{|c|c|c|c|c|c|c|}
\hline Brain regions & Voxels & $\mathrm{BA}$ & \multicolumn{3}{|c|}{ MNI coordinates } & $\mathrm{F} / \mathrm{T}$ value \\
\hline \multicolumn{7}{|l|}{ ANOVA } \\
\hline Cerebelum-Crus I-L & 32 & - & -36 & -48 & -36 & 6.4958 \\
\hline Insula-R & 46 & 47 & 33 & 24 & 3 & 10.4319 \\
\hline Frontal-Sup-L & 37 & 10 & -15 & 66 & 21 & 8.9487 \\
\hline Frontal-Inf-Tri-L & 70 & 48 & -42 & 15 & 27 & 12.9862 \\
\hline Frontal-Mid-R & 34 & 8 & 27 & 27 & 48 & 9.0315 \\
\hline \multicolumn{7}{|l|}{$\mathrm{AD}$ vs. $\mathrm{MCl}$} \\
\hline Frontal-Inf-Tri-L & 61 & 48 & -45 & 18 & 27 & -4.9329 \\
\hline \multicolumn{7}{|l|}{$A D$ vs. NC } \\
\hline Cerebelum-Crus I-L & 31 & - & -36 & -48 & -36 & 3.1791 \\
\hline Insula-R & 26 & 47 & 30 & 27 & 0 & -3.5715 \\
\hline Frontal-Inf-Tri-L & 53 & 48 & -42 & 15 & 27 & -4.8272 \\
\hline Frontal-Sup-R & 34 & 8 & 24 & 27 & 51 & -3.9857 \\
\hline \multicolumn{7}{|l|}{$\mathrm{MCl}$ vs. NC } \\
\hline Insula-R & 28 & 47 & 30 & 24 & 3 & -4.1713 \\
\hline
\end{tabular}

FCD, functional connectivity density; AD, Alzheimer's disease; $\mathrm{MCl}$, mild cognitive impairment; NC, normal control group; MNI, Montreal Neurological Institute; BA, Brodmann area; ANOVA, analysis of variance; L, left; R, right; Inf, inferior; Sup, superior; Mid, middle; Tri, triangular.

a stronger short-range FCD value than the late-stage of $\mathrm{AD}$. Stronger short-range FCD may reduce the progression of $\mathrm{AD}$, which can help early diagnosis and treatment. The increased short-range FCD could be a compensation mechanism in the left inferior frontal gyrus for cognitive function during MCI progression to $\mathrm{AD}$ (24).

\section{FCD changes in the cerebellum}

Earlier studies have suggested aberrant FCD in the cerebellum indicative of susceptibility to cognition decline. We observed increased short- and long-range FCD in the cerebellum involved in social cognition function $(25,26)$. Patients with a cerebellum-specific disease have been shown to present impaired executive functions and abstract reasoning (27), consistent with the impairment observed in patients with cognition decline. Consistent with our findings, a meta-analysis on MRI studies on whole-brain function reported a significant association of activation of the posterior cerebellar hemispheres, especially the Crus I (28). As showed by several fMRI studies, the cerebellar is involved in working memory tasks (29). Working memory impairment is a significant cognition decline symptom in patients with $\mathrm{AD}$ and $\mathrm{MCI}$ (30). Computational modeling of neural dynamics has shown that the cerebellum is the degree of centrality structure for sensorimotor integration and motor preparation; the cerebellar exclusion could have affect evidence in cognitive processes (31). In humans, there is accumulating evidence showing that the forebrain regions receive input from the lateral posterior cerebellum; cerebellum disruption results in impaired working memory (32).

\section{FCD changes in the frontal lobe}

Compared with the $\mathrm{AD}$ and $\mathrm{NC}$ groups, the MCI group showed a significantly higher short-range FCD value 

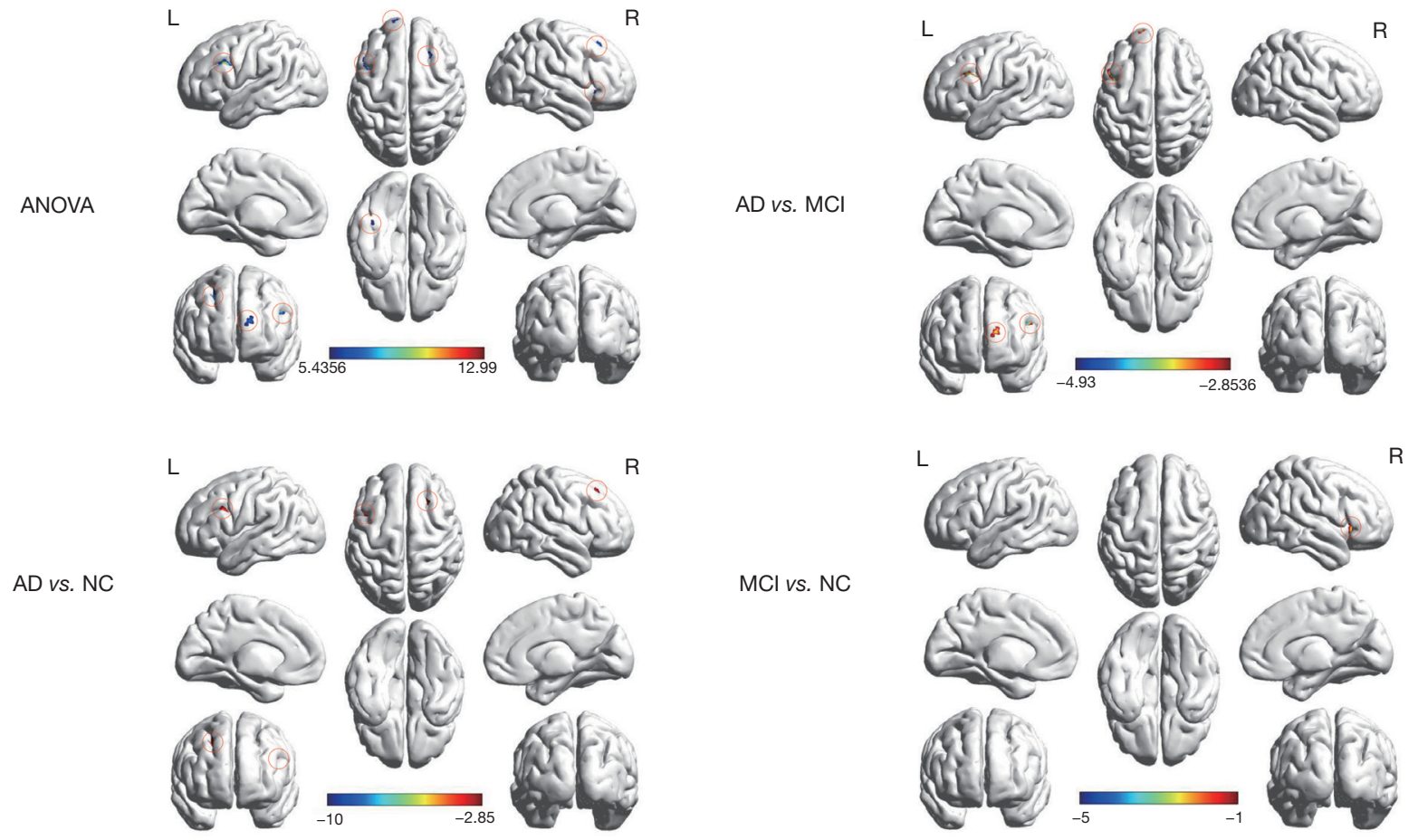

Figure 2 Spatial distribution of long-range FCD in the AD, MCI, and NC and the statistical among-group differences. The among-group differences were corrected using AlphaSim ( $\mathrm{P}<0.005$, a combined threshold of $\mathrm{P}<0.05$ with a minimum cluster size of 25 voxels). ANOVA, Analysis of variance; AD, Alzheimer's Disease; MCI, mild cognitive impairment; NC, normal control group; L, left; R, right; FCD, functional connectivity density.

and lower long-range FCD value in the frontal gyrus, reflecting the neuroimaging endophenotypes patients with $\mathrm{AD}$ and MCI (33). It may be crucially involved in pathology development and its trajectory in patients with $\mathrm{AD}$ and MCI. Previous studies have reported increasing $\mathrm{FC}$ in the frontal lobes in MCI, maintaining episodic memory (34). A previous study reported impaired activation and connectivity within specific elements of the frontal-medical temporal lobe circuit, which is crucially involved in component processes of episodic memory and cognition (35). A recent study reported increasing FC in the frontal lobe in individuals with MCI after 12 weeks of exercise training (36). Direct evidence from intraoperative stimulation and diffusion tractography showed that the inferior frontal gyrus is crucially involved in an interference, which could help maintain cognitive control processes (37). A synchronizing rhythmic brain stimulation study showed a preferential increase in frontotemporal regions and rapid improvement in working memory performance, after 25 -minute stimulation (38). Further well-designed studies precisely explore the effect of FCD in the left inferior frontal gyrus and right middle frontal gyrus by using multiple techniques, including deep brain stimulation, transcranial direct current stimulation, and repetitive transcranial magnetic stimulation. Those further studies may help us understand the FCD in AD pathology.

\section{Correlation analysis and MMSE effect}

We found that it significantly correlated the connectivity strength of the brain regions with altered FC with MMSE scores in patients with AD and MCI. These findings suggest that altered FC of seeds based on clusters with abnormal FCD over the entire brain reflects intrinsic dysfunction changes in patients with $\mathrm{AD}$ and MCI, which might change according to the clinical state. These results suggest that aberrant FCD is an independent $\mathrm{AD}$ and MCI characteristic. However, there is a need for further studies on the neuro-mechanism to elucidate the reciprocity between abnormal FCD changes and neuropsychological variables in patients with cognition decline. In further study, this relationship between the FCD and neuropsychological variables can be used in early diagnosis and predict the 

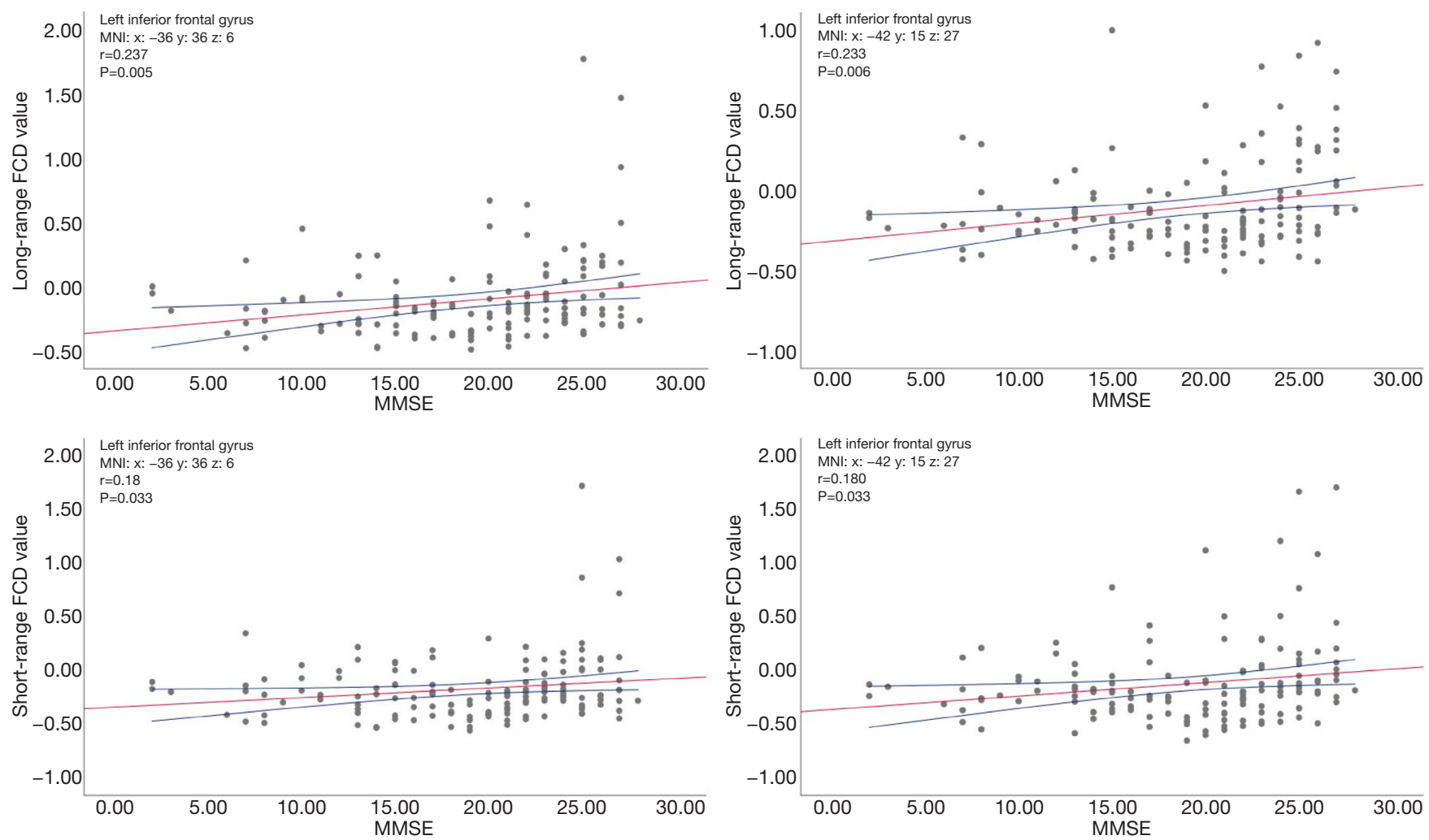

Figure 3 There was a positive correlation between MMSE scores and long-range FCD in the left inferior frontal gyrus. There was a positive correlation of short-range FCD in the left inferior frontal gyrus with MMSE scores. MMSE, Mini-mental State Examination; FCD, functional connectivity density.

development of cognitive function.

\section{Conclusions}

We observed weakened long-range FCD and enhanced short-range FCD, which could be the mechanism and compensation of the loss of cognitive function. These findings could improve the understanding of $\mathrm{AD}$ and $\mathrm{MCI}$ pathology and supply a potential neuroimaging marker for brain dysfunction in $\mathrm{AD}$ and MCI.

\section{Limitations}

There are several limitations to this study. First, the neural compensation observed in this cross-sectional study should be interpreted with caution. Second, the premise of functional computing connections is to assume that the time series still is constant; it could not optimally reflect the dynamic nature of brain activity, the sliding window correlation analysis would be used for evaluating dynamic functional connectivity in further study. Third, the limited clinical data of participants could restrict the statistical power for characterizing the neuroimaging evidence of a cognitive decline in patients with $\mathrm{AD}$ and $\mathrm{MCI}$, more $\mathrm{AD}$ related assessment scale need to be collected including the activities of daily living, behavioral and psychological symptoms in further study.

\section{Acknowledgments}

We thank all the health workers and patients who contributed to our work and took part in the present questionnaire survey.

Funding: This pilot study was funded by the National Natural Science Foundation of China (No. 81771158), the General Project of the Department of Science and Technology of Zhejiang Province (2020358406), Science Foundation of the Health Commission of Zhejiang Province (Nos. 2019321345). 


\section{Footnote}

Reporting Checklist: The authors have completed the MDAR reporting checklist. Available at http://dx.doi.org/10.21037/ atm-20-7019

Data Sharing Statement: Available at http://dx.doi. org/10.21037/atm-20-7019

Conflict of Interest: All authors have completed the ICMJE uniform disclosure form (available at http://dx.doi. org/10.21037/atm-20-7019). The authors have no conflicts of interest to declare.

Ethical Statement: The authors are accountable for all aspects of the work in ensuring that questions related to the accuracy or integrity of any part of the work are appropriately investigated and resolved. This study was approved by the Ethics Committee of Zhejiang Provincial People's Hospital (No. 2012KY002) and was performed following the Declaration of Helsinki (as revised in 2013). Informed consent was taken from all the patients.

Open Access Statement: This is an Open Access article distributed in accordance with the Creative Commons Attribution-NonCommercial-NoDerivs 4.0 International License (CC BY-NC-ND 4.0), which permits the noncommercial replication and distribution of the article with the strict proviso that no changes or edits are made and the original work is properly cited (including links to both the formal publication through the relevant DOI and the license). See: https://creativecommons.org/licenses/by-nc-nd/4.0/.

\section{References}

1. Scheltens P, Blennow K, Breteler MM, et al. Alzheimer's disease. Lancet 2016;388:505-17.

2. Mortamais M, Ash JA, Harrison J, et al. Detecting cognitive changes in preclinical Alzheimer's disease: A review of its feasibility. Alzheimers Dement 2017;13:468-92.

3. Sun Y, Lin J, Zhang L. The application of weighted gene co-expression network analysis in identifying key modules and hub genes associated with disease status in Alzheimer's disease. Ann Transl Med 2019;7:800.

4. Li H, Habes M, Wolk DA, et al. A deep learning model for early prediction of Alzheimer's disease dementia based on hippocampal magnetic resonance imaging data. Alzheimers Dement 2019;15:1059-70.
5. Gaubert S, Raimondo F, Houot M, et al. EEG evidence of compensatory mechanisms in preclinical Alzheimer's disease. Brain 2019;142:2096-112.

6. Firth NC, Primativo S, Marinescu RV, et al. Longitudinal neuroanatomical and cognitive progression of posterior cortical atrophy. Brain 2019;142:2082-95.

7. Marcos Dolado A, Gomez-Fernandez C, Yus Fuertes $M$, et al. Diffusion Tensor Imaging Measures of Brain Connectivity for the Early Diagnosis of Alzheimer's Disease. Brain Connect 2019;9:594-603.

8. Sarpal DK, Robinson DG, Lencz T, et al. Antipsychotic treatment and functional connectivity of the striatum in firstepisode schizophrenia. JAMA Psychiatry 2015;72:5-13.

9. Feng Q, Wang M, Song Q, et al. Correlation Between Hippocampus MRI Radiomic Features and Resting-State Intrahippocampal Functional Connectivity in Alzheimer's Disease. Front Neurosci 2019;13:435.

10. Shokri-Kojori E, Tomasi D, Wiers CE, et al. Alcohol affects brain functional connectivity and its coupling with behavior: greater effects in male heavy drinkers. Mol Psychiatry 2017;22:1185-95.

11. Lei X, Liao Y, Zhong M, et al. Functional Connectivity Density, Local Brain Spontaneous Activity, and Their Coupling Strengths in Patients With Borderline Personality Disorder. Front Psychiatry 2018;9:342.

12. Kong D, Liu R, Song L, et al. Altered Long- and ShortRange Functional Connectivity Density in Healthy Subjects After Sleep Deprivations. Front Neurol 2018;9:546.

13. Tomasi D, Volkow ND. Reduced Local and Increased Long-Range Functional Connectivity of the Thalamus in Autism Spectrum Disorder. Cereb Cortex 2019;29:573-85.

14. Alderson T, Kehoe E, Maguire L, et al. Disrupted Thalamus White Matter Anatomy and Posterior Default Mode Network Effective Connectivity in Amnestic Mild Cognitive Impairment. Front Aging Neurosci 2017;9:370.

15. Wang J, Wang X, Xia M, et al. GRETNA: a graph theoretical network analysis toolbox for imaging connectomics. Front Hum Neurosci 2015;9:386.

16. Tomasi D, Volkow ND. Functional connectivity density mapping. Proceedings of the National Academy of Sciences of the United States of America 2010;107:9885-90.

17. Spadone S, Della Penna S, Sestieri C, et al. Dynamic reorganization of human resting-state networks during visuospatial attention. Proc Natl Acad Sci U S A 2015;112:8112-7.

18. He Y, Chen ZJ, Evans AC. Small-world anatomical networks in the human brain revealed by cortical thickness 
from MRI. Cereb Cortex 2007;17:2407-19.

19. Buckner RL, Sepulcre J, Talukdar T, et al. Cortical hubs revealed by intrinsic functional connectivity: mapping, assessment of stability, and relation to Alzheimer's disease. J Neurosci 2009;29:1860-73.

20. Beucke JC, Sepulcre J, Talukdar T, et al. Abnormally high degree connectivity of the orbitofrontal cortex in obsessive-compulsive disorder. JAMA Psychiatry 2013;70:619-29.

21. Muhle-Karbe PS, Derrfuss J, Lynn MT, et al. CoActivation-Based Parcellation of the Lateral Prefrontal Cortex Delineates the Inferior Frontal Junction Area. Cereb Cortex 2016;26:2225-41.

22. Nissim NR, O'Shea AM, Bryant V, et al. Frontal Structural Neural Correlates of Working Memory Performance in Older Adults. Front Aging Neurosci 2017;8:328.

23. Slattery CF, Agustus JL, Paterson RW, et al. The functional neuroanatomy of musical memory in Alzheimer's disease. Cortex 2019;115:357-70.

24. Wang J, Liu J, Wang Z, et al. Dysfunctional interactions between the default mode network and the dorsal attention network in subtypes of amnestic mild cognitive impairment. Aging (Albany NY) 2019;11:9147-66.

25. Wagner MJ, Luo L. Neocortex-Cerebellum Circuits for Cognitive Processing. Trends Neurosci 2020;43:42-54.

26. Keefe MG, Nowakowski TJ. Evolutionary Expansion of Human Cerebellar Germinal Zones. Trends Neurosci 2020;43:75-7.

27. Schmahmann JD, Sherman JC. The cerebellar cognitive affective syndrome. Brain 1998;121:561-79.

28. Van Overwalle F, Baetens K, Mariën P, et al. Social cognition and the cerebellum: a meta-analysis of over 350 fMRI studies. Neuroimage 2014;86:554-72.

29. Peterburs J, Blevins LC, Sheu YS, et al. Cerebellar contributions to sequence prediction in verbal working

Cite this article as: Mao Y, Liao Z, Liu X, Li T, Hu J, Le D, Pei Y, Sun W, Lin J, Qiu Y, Zhu J, Chen Y, Qi C, Su H, Yu E. Disrupted balance of long and short-range functional connectivity density in Alzheimer's disease (AD) and mild cognitive impairment (MCI) patients: a resting-state fMRI study. Ann Transl Med 2021;9(1):65. doi: 10.21037/atm-207019 memory. Brain Struct Funct 2019;224:485-99.

30. Kumar S, Zomorrodi R, Ghazala Z, et al. Extent of Dorsolateral Prefrontal Cortex Plasticity and Its Association With Working Memory in Patients With Alzheimer Disease. JAMA Psychiatry 2017;74:1266-74.

31. Guell X, Gabrieli JDE, Schmahmann JD. Triple representation of language, working memory, social and emotion processing in the cerebellum: convergent evidence from task and seed-based resting-state fMRI analyses in a single large cohort. Neuroimage 2018;172:437-49.

32. Bostan AC, Strick PL. The basal ganglia and the cerebellum: nodes in an integrated network. Nat Rev Neurosci 2018;19:338-50.

33. Franzmeier N, Hartmann JC, Taylor ANW, et al. Left Frontal Hub Connectivity during Memory Performance Supports Reserve in Aging and Mild Cognitive Impairment. J Alzheimers Dis 2017;59:1381-92.

34. Franzmeier N, Duering $M$, Weiner M, et al. Left frontal cortex connectivity underlies cognitive reserve in prodromal Alzheimer disease. Neurology 2017;88:1054-61.

35. Guo JY, Ragland JD, Carter CS. Memory and cognition in schizophrenia. Mol Psychiatry 2019;24:633-42.

36. Chirles TJ, Reiter K, Weiss LR, et al. Exercise Training and Functional Connectivity Changes in Mild Cognitive Impairment and Healthy Elders. J Alzheimers Dis 2017;57:845-56.

37. Puglisi G, Howells H, Sciortino T, et al. Frontal pathways in cognitive control: direct evidence from intraoperative stimulation and diffusion tractography. Brain 2019;142:2451-65.

38. Reinhart RMG, Nguyen JA. Working memory revived in older adults by synchronizing rhythmic brain circuits. Nat Neurosci 2019;22:820-7.

(English Language Editor: J. Chapnick) 